\title{
The use of representativity theory in the depletion calculations of SFR blankets
}

\author{
Jean-François Lebrat* , Jean Tommasi \\ CEA, DEN, Cadarache, DER, SPRC, F-13-108 Saint-Paul-Lez-Durance, France
}

\begin{abstract}
The analysis of the DOUBLON experiment has provided C/E's for several isotopic ratios in the first and second rows of the radial blanket after an irradiation in the PHENIX Sodium Fast Reactor. We have used this as a basis for the validation of the DARWIN-2.3 (Tsilanizara et al., 2000) package for the depletion calculation in the radial blankets of SFR's (Lebrat et al., 2015). Unfortunately, no measurements have been performed in the upper and lower axial blankets of the fissile assemblies. Of course we expect our calculations to be reliable in these zones as well, as the initial compositions, nuclear reactions and neutron spectra are very similar. The representativity theory appeared to be the appropriate tool to correlate the available experimental information in the radial blanket to our calculations in the axial zones. Indeed, the representativity factors that we calculate between the final ${ }^{239} \mathrm{Pu} /{ }^{238} \mathrm{U}$ ratio in the radial and axial fertile zones are very close to 1 . Hence, the measurements performed in the radial zone can help us "correct" the calculation in the axial blanket and reduce its a-priori uncertainty due to nuclear data. The estimated uncertainty reduction can reach a factor 5 , since the experimental uncertainty is low. The ${ }^{240} \mathrm{Pu} /{ }^{238} \mathrm{U}$ ratio is studied with a similar method.
\end{abstract}

\section{Introduction}

The analysis of the DOUBLON experiment has provided C/E's for several isotopic ratios in the first and second rows of the radial blanket after an irradiation in the PHENIX Sodium Fast Reactor. We have used this as a basis for the validation of the DARWIN2.3 (Tsilanizara et al., 2000) package for the depletion calculation in the radial blankets of SFR's (Lebrat et al., 2015). Unfortunately, no measurements have been performed in the upper and lower axial blankets of the fissile assemblies. Of course we expect our calculations to be reliable in these zones as well, as the initial compositions, nuclear reactions and neutron spectra are very similar. The representativity theory appeared to be the appropriate tool to correlate the available experimental information in the radial blanket to our calculations in the axial zones.

\section{Definition of the problem}

The parameter we have chosen to study is the final ${ }^{239} \mathrm{Pu} /{ }^{238} \mathrm{U}$ concentrations ratio in the blanket after irradiation and cooling. Indeed, the ${ }^{239} \mathrm{Pu}$ is the main isotope produced in the fertile zones,

\footnotetext{
* Corresponding author.

E-mail address: jean-francois.lebrat@cea.fr (J.-F. Lebrat).
}

whereas the amount of ${ }^{238} \mathrm{U}$ is almost constant. This makes the final ${ }^{239} \mathrm{Pu} /{ }^{238} \mathrm{U}$ ratio a good measurement of the "burnup" in the blankets.

The ${ }^{239} \mathrm{Pu}$ is produced in the fertile zones by a capture reaction on the initial ${ }^{238} \mathrm{U}$ (via the ${ }^{239} \mathrm{~Np}$ ), so the final amount of ${ }^{239} \mathrm{Pu}$ depends on the neutron spectrum (via the cross sections) and the decay constants of ${ }^{239} \mathrm{~Np}$ and ${ }^{239} \mathrm{Pu}$. These dependencies are quantified by relative sensitivity coefficients: they measure the response of an integral parameter to a variation of a nuclear data. They are calculated by using the perturbation theory (Kallfelz et al., 1997; Williams, 1978, 1986) and can be written in our case:

$S_{\sigma}=\frac{\sigma}{N} \times \frac{\partial N}{\partial \sigma}$

where $S$ is a vector with $N_{\text {isotopes }} \times N_{\text {reactions }} \times N_{\text {groupes }}$ entries, to which we must add the decay constants of the radioactive isotopes involved.

The sensitivity coefficients are computed for each isotope, each reaction and each of the 33 energy groups by the MECCYCO module of the ERANOS package (Ruggieri et al., 2006). This program performs adjoint multigroup evolution calculations, in order to obtain adjoint concentrations (i.e. importances). The relative sensitivities on the ${ }^{239} \mathrm{Pu} /{ }^{238} \mathrm{U}$ ratio are then obtained by a simple relationship: 
$S_{\left(\frac{P u 239}{U 238}\right)}^{\sigma}=\frac{\sigma}{\left(\frac{P u 239}{U 238}\right)} \times \frac{\partial}{\partial \sigma}\left(\frac{P u 239}{U 238}\right)$

$S_{\left(\frac{P u 239}{U 238}\right)}^{\sigma}=\frac{\sigma}{\left(\frac{P u 239}{U 238}\right)}$

$$
\times\left(\frac{1}{U 238}\right)^{2}\left\lfloor U 238 \times \frac{\partial}{\partial \sigma}\left(\text { Pu239) }- \text { Pu239 } \times \frac{\partial}{\partial \sigma}(U 238)\right\rfloor\right.
$$

$S_{\left(\frac{P u 239}{U 238}\right)}^{\sigma}=\frac{\sigma}{P u 239} \times \frac{\partial}{\partial \sigma}(P u 239)-\frac{\sigma}{U 238} \times \frac{\partial}{\partial \sigma}(U 238)$

$S_{\left(\frac{P u 239}{U 238}\right)}^{\sigma}=S_{P u 239}^{\sigma}-S_{U 238}^{\sigma}$

where "239 Pu" and "238 U” are the final concentrations of each isotope. We observe that the relative sensitivity on the isotopic ratio is simply the difference of the individual relative sensitivities.

Once the relative sensitivity coefficients are calculated, the uncertainty $\varepsilon$ (in \%) on any integral (in term of energy) parameter is then given by:

$\varepsilon=\sqrt{S^{t} D S}$

where $D$ is the variance-covariance matrix of the nuclear data (the cross entries are in $[\%]^{2}$ ).

The representativity coefficient (Orlov, 1980) is usually used to compare a reactor concept with an experiment (Dos Santos et al., 2013a). We use it here in order to give the "similarity", the «correlation $\gg$ between the ${ }^{239} \mathrm{Pu} /{ }^{238} \mathrm{U}$ ratios in two different zones 1 and 2 of a reactor by using their respective sensitivity vectors to nuclear data $S_{1}$ and $S_{2}$ :

$r_{1,2}=\frac{S_{1}^{t} D S_{2}}{\sqrt{S_{1}^{t} D S_{1}} \sqrt{S_{2}^{t} D S_{2}}}$

$D$ is the covariance matrix of the cross sections involved; we use the COMAC-V0 release (Archier et al., 2014) in a 33 energy groups mesh, based on the JEFF-3.1.1 library (JEFF-3.1.1, 0000).

Physically, $r$ represents how much information the two zones "have in common" for a given parameter.

- If the two sensitivity vectors are identical, then $r$ is maximal $r=1$,

- The more different the sensitivities are, the more $r$ decreases,

- If $r=0$, it means that the productions of ${ }^{239} \mathrm{Pu}$ in the two zones are not correlated at all.

In our case, the covariance matrix must take into account the capture (c) and fission (f) of ${ }^{238} \mathrm{U},{ }^{239} \mathrm{~Np}$ and ${ }^{239} \mathrm{Pu}$. The two last diagonal entries $D_{199,199}$ and $D_{200,200}$ account for the decay of ${ }^{239} \mathrm{~Np}$ and ${ }^{239} \mathrm{Pu}$ and finally $\mathrm{D}$ can be written as:

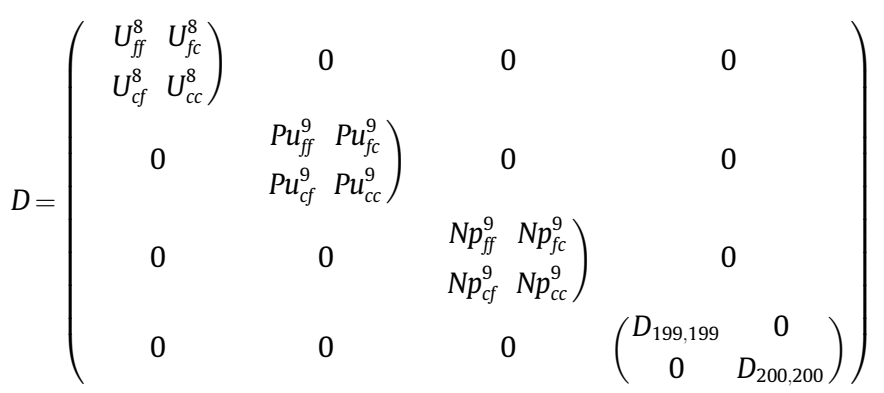

This matrix represents the uncertainties and correlations, which cover the different groups as well as the different reactions. For instance, $U_{f f}^{8}$ is a $33 * 33$ matrix, whose:
- Diagonal entries are the squares of the relative uncertainties (in $\%)$ of the ${ }^{238} \mathrm{U}$ fission cross section for each energy group,

- The non-diagonal entries are the correlations between the different energy groups for the ${ }^{238} \mathrm{U}$ fission reaction.

The other square blocks $U_{f c}^{8}, U_{c f}^{8}$ and $U_{c c}^{8}$ are defined the same way and similar matrixes are defined for the other isotopes. It must be emphasized that $D$ contains correlations between the various reactions for a given isotope, but not yet between different isotopes.

The two last diagonal entries $D_{199,199}$ and $D_{200,200}$ are the squares of the relative uncertainties on the decay constants $\lambda$ of ${ }^{239} \mathrm{~Np}$ ( $\beta$-decay) and ${ }^{239} \mathrm{Pu}$ ( $\alpha$ decay). They are deduced from the halflifes $T$ available in JEFF-3.1.1 (0000) by the following formula:

$\left(\frac{\delta \lambda}{\lambda}\right)^{2}=\left(\frac{\delta T}{T}\right)^{2}$

The values of $D_{199,199}$ and $D_{200,200}$ are calculated in Table 1 .

So finally $D$ will have $(6 \times 33+2)^{2}=200^{2}$ entries.

\section{Application to our study}

For our application, the above parameters are calculated in the positions of the PHENIX reactor that are represented on Fig. 1:

- At the core midplane of the inner fuel assembly (FUEL) located in position $18 / 20$,

- At mid-height of the lower (CAI) and upper (CAS) axial blankets of the same fuel assembly,

- In the first row of the radial blanket (FEF), at the core midplane of the assembly $24 / 13$,

- In the second row of the radial blanket (FEG), at the core midplane of the assembly 24/12.

The 42.8 EFPD's of the cycle 10 of the PHENIX reactor are computed with the ERANOS package and its integrated depletion module MECCYCO, with the same calculation route that was used for the interpretation of the TRAPU and DOUBLON irradiations (Lebrat et al., 2015). The sensitivities are evaluated in "fresh" fertile blankets composed of natural (for the CAI) or depleted (for the CAS, FEF and FEG) Uranium.

Fig. 2 shows the 33 energy group neutron spectrum obtained in the different zones of the reactor that we have studied; it is very similar in the various fertile zones and much "harder" in the fuel.

The same comment can be made on the sensitivity profiles shown on Figs. 3-6; to make the comparison easier on these figures, each sensitivity coefficients is normalized by its integral over energy. By definition, the normalized relative sensitivity coefficients are all positive.

Once these sensitivities are calculated, we use MATLAB to perform the calculation of the representativity factors between two zones 1 and 2 as defined by Eq. (4). The results are detailed in Table 2, as well as the uncertainties due to nuclear data $\varepsilon$ that are defined by Eq. (3).

Table 2 shows that the representativity factors between any two of the various fertile zones are very close to 1 . Of course, this was very predictable because the sensitivity profiles are very close

Table 1

Variances of the ${ }^{239} \mathrm{~Np}$ and ${ }^{239} \mathrm{Pu}$ decay constants.

\begin{tabular}{llll}
\hline & $\mathrm{T}(\mathrm{s})$ & $\delta \mathrm{T}(\mathrm{s})$ & $\left(\frac{\delta \mathrm{T}}{\mathrm{T}}\right)^{2}\left(\%^{2}\right)$ \\
\hline${ }^{239} \mathrm{~Np}(\beta-)$ & $2.0347 \cdot 10^{5}$ & $3.456 \cdot 10^{2}$ & $2.885 \cdot 10^{-2}$ \\
${ }^{239} \mathrm{Pu}(\alpha)$ & $7.6094 \cdot 10^{11}$ & $3.4713 \cdot 10^{8}$ & $2.081 \cdot 10^{-3}$ \\
\hline
\end{tabular}




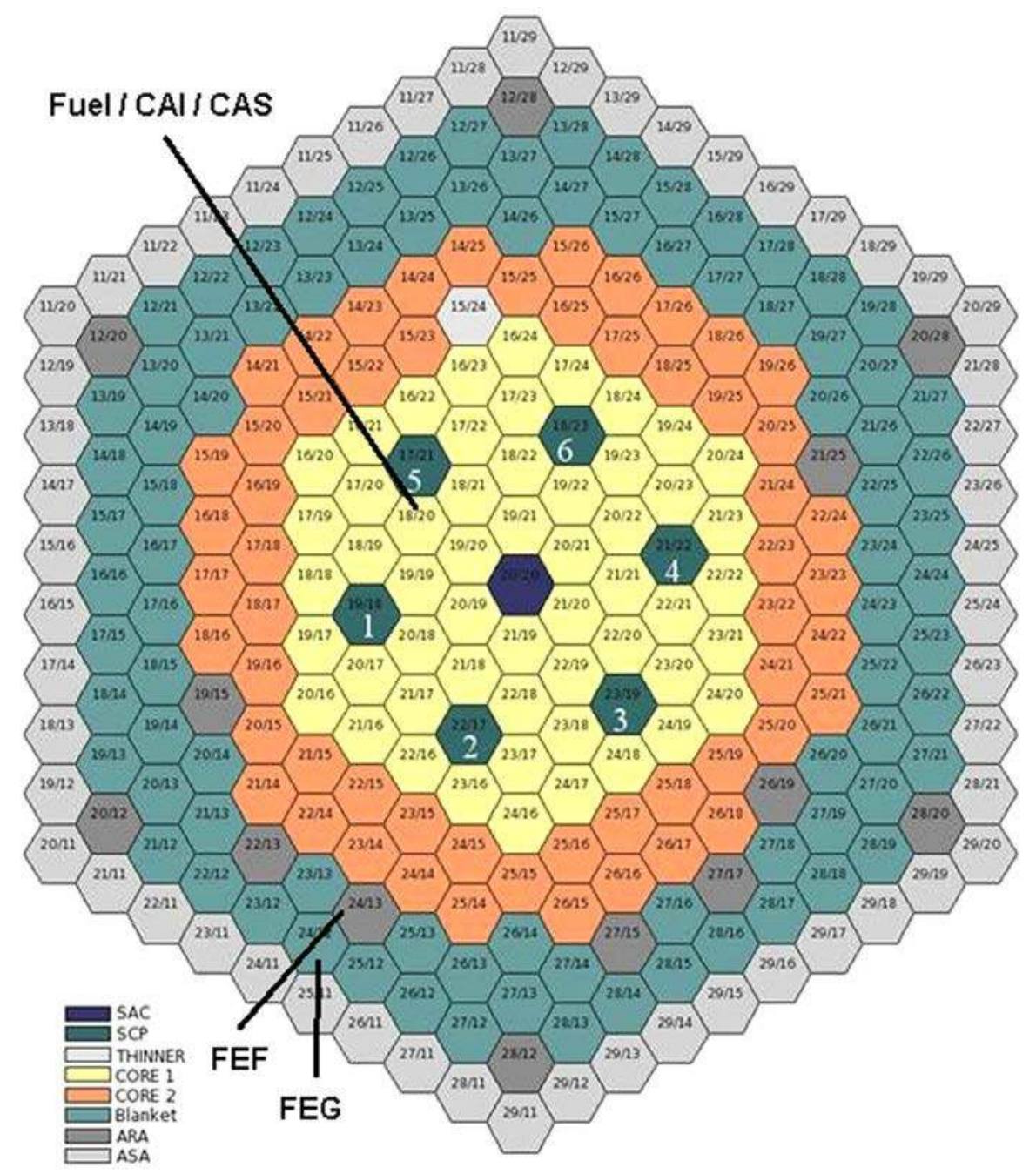

Fig. 1. Position of the fuel and fertile zone in the PHENIX reactor.

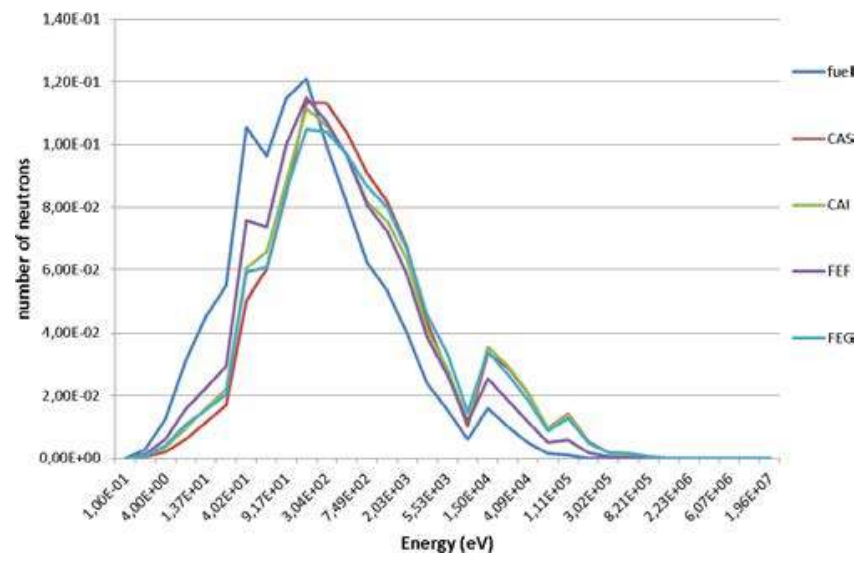

Fig. 2. 33 groups normalized neutron energy spectrum calculated by ERANOS in the various zones of the reactor.

to each other: we are comparing similar nuclear reactions in zones with similar initial compositions and very close neutron spectra.

It is important to note that, whilst the formalism of representativity and transposition only considers its contribution $\varepsilon$ due to the nuclear data, the total uncertainty on the calculated ${ }^{239} \mathrm{Pu} /{ }^{238} \mathrm{U}$ ratio has other contributions:

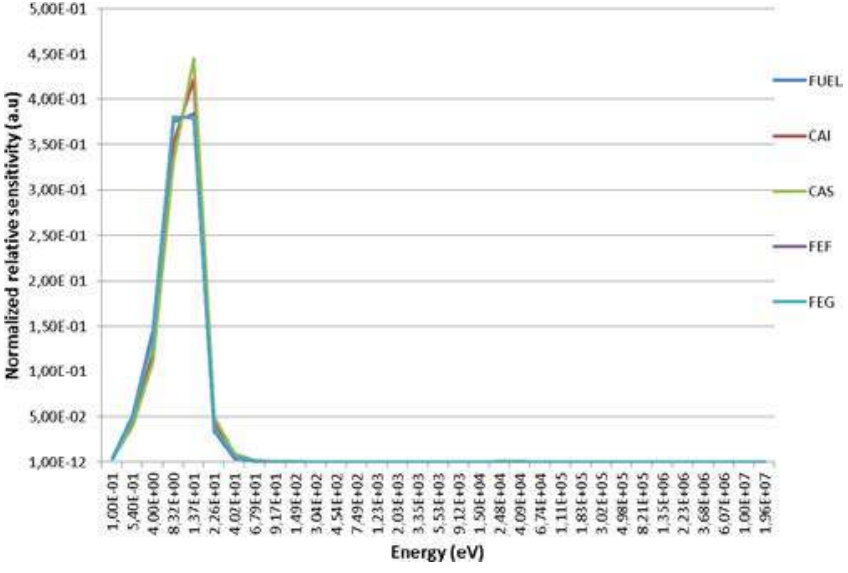

Fig. 3. Normalized relative sensitivity of the ${ }^{239} \mathrm{Pu} /{ }^{238} \mathrm{U}$ ratio to the ${ }^{238} \mathrm{U}$ fission cross section as a function of energy.

- In the case of DOUBLON, a fluence adjustment of $+1 \%$ is necessary for the calculation to match the measured ${ }^{148} \mathrm{Nd} /{ }^{238} \mathrm{U}$ ratio (Lebrat et al., 2015). The uncertainty on the fission yield of ${ }^{148} \mathrm{Nd}$ associated to ${ }^{239} \mathrm{Pu}$ being $1.70 \%$ (99\% of the ${ }^{148} \mathrm{Nd}$ comes from fissions on the ${ }^{239} \mathrm{Pu}$ ), this uncertainty will reflect on the calculated ${ }^{239} \mathrm{Pu} /{ }^{238} \mathrm{U}$ ratio. 


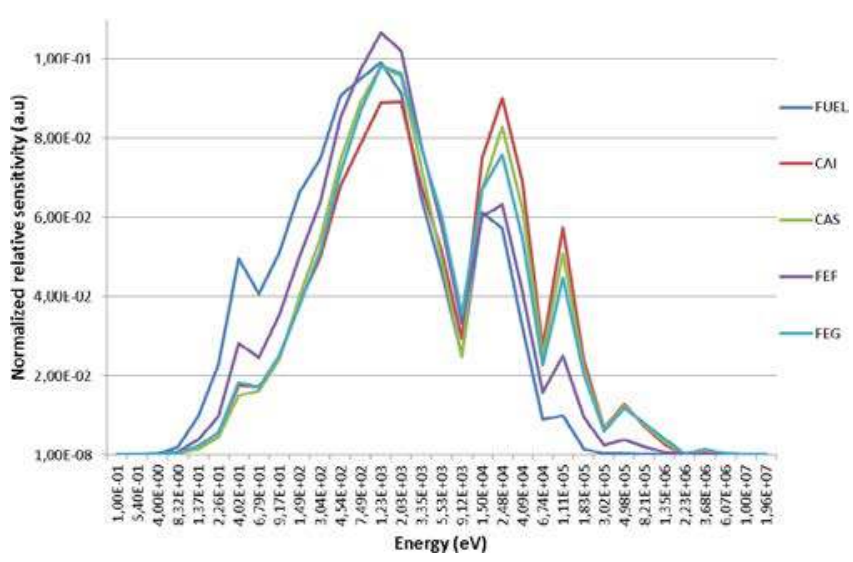

Fig. 4. Normalized relative sensitivity of the ${ }^{239} \mathrm{Pu} /{ }^{238} \mathrm{U}$ ratio to the ${ }^{238} \mathrm{U}$ capture cross section as a function of energy.

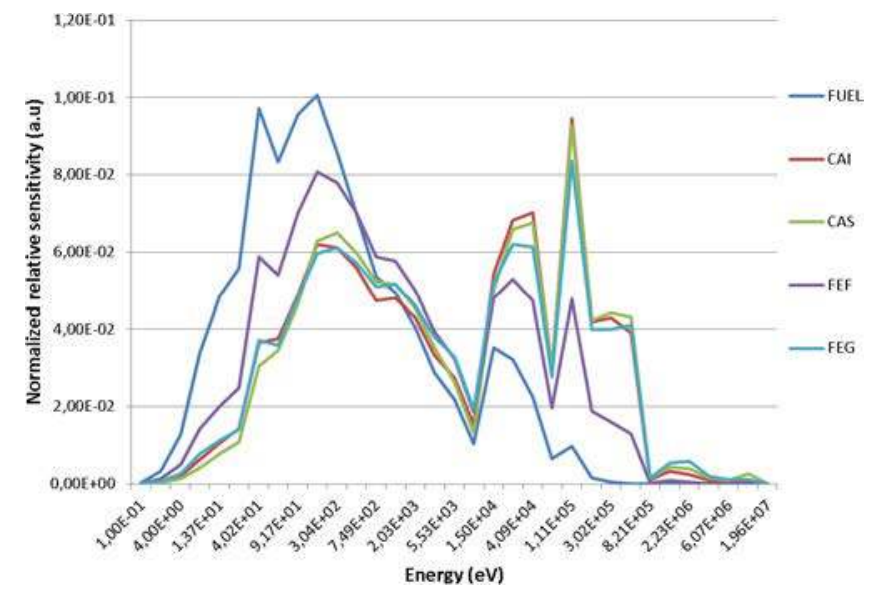

Fig. 5. Normalized relative sensitivity of the ${ }^{239} \mathrm{Pu} /{ }^{238} \mathrm{U}$ ratio to the ${ }^{239} \mathrm{Pu}$ fission cross section as a function of energy.

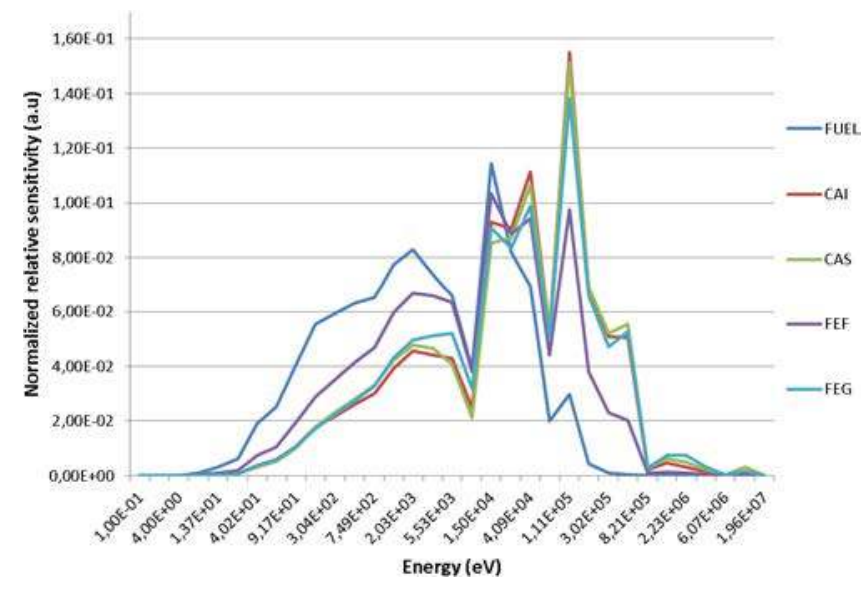

Fig. 6. Normalized relative sensitivity of the ${ }^{239} \mathrm{Pu} /{ }^{238} \mathrm{U}$ ratio to the ${ }^{239} \mathrm{Pu}$ capture cross section as a function of energy.

- The «technological» uncertainty accounts for the difference between the "reality" of the reactor and the simulation we perform in our calculation codes: isotopic concentrations, temperature, thickness or pitch tolerances, etc... this uncertainty is estimated to be around $0.1 \%(100 \mathrm{pcm})$ on the multiplication factor $k_{\text {eff }}$ in our case (Dos Santos et al., 2013b), so it is probably much lower on the calculated ${ }^{239} \mathrm{Pu} /{ }^{238} \mathrm{U}$ ratio we consider here.
Table 2

Representativity factors for the depletion calculation of the ${ }^{239} \mathrm{Pu} /{ }^{238} \mathrm{U}$ ratio and apriori uncertainties due to nuclear data.

\begin{tabular}{llll}
\hline $1 / 2$ & $\mathrm{r}_{1,2}$ & $\varepsilon_{1}$ & $\varepsilon_{2}$ \\
\hline FEF/CAS & 0.9927 & $3.45 \%$ & $2.74 \%$ \\
FEG/CAS & 0.9995 & $2.80 \%$ & $2.74 \%$ \\
FEF/CAI & 0.9926 & $3.45 \%$ & $2.67 \%$ \\
FEG/CAI & 0.9989 & $2.80 \%$ & $2.67 \%$ \\
\hline
\end{tabular}

\section{Transposition and uncertainty reduction}

As we have mentioned, the high representativity factors we have calculated can help us determine - for instance - to what extend the measurements performed in the first raw of the radial blanket FEF can "correct" the calculation in the lower axial blanket CAI. This is performed with the following formula (Gandini, 1983):

$\frac{C_{C A I}^{\prime}-C_{C A I}}{C_{C A I}}=\frac{E-C_{F E F}}{C_{F E F}}\left(r \frac{\varepsilon_{C A I}}{\varepsilon_{F E F}} \frac{1}{1+\left(\frac{\delta E}{\varepsilon_{F E F}}\right)^{2}}\right)$

where $C_{C A I}$ is the ERANOS/DARWIN calculation result and $\varepsilon_{C A I}$ the associated uncertainty due to nuclear data. $C_{C A I}^{\prime}$ is the calculation "corrected" by the experimental information in the FEF zone and the associated calculation, via the representativity factor $r$ between CAI and FEF.

As an illustration, let us consider the central pin of assembly FEF79 that was studied in the DOUBLON experiment. For this pin, $\mathrm{E}=0.03131 \pm 1.7 \%, C_{F E F}=0.031449$ and from Table 2 we obtain $r=0.9926, \varepsilon_{F E F}=3.45 \%$ and $\varepsilon_{C A I}=2.67 \%$. The experimental uncertainty is equal to $0.5 \%$ (Lebrat et al., 2015).

With Eq. (7), we are then able to calculate: $\frac{\frac{C}{C A I}_{C C_{C A I}}^{\prime} C_{C A I}}{C_{C A I}}=-0.0033$.

This means that we have a minor correction of $-0.33 \%$ on the a priori calculation in the CAI due to the measurement performed in the FEF. In our case, this correction is small because:

- The $\frac{E-C_{F E F}}{C_{F E F}}$ ratio is small, meaning that was have a good $C$ vs $E$ agreement in the radial blanket,

- The a-priori uncertainties due to nuclear data $\varepsilon_{C A I}$ and $\varepsilon_{F E F}$ have the same order of magnitude.

The high representativity between the radial and axial blankets calculations shows that our a-priori calculation in the axial blanket is reliable and that the correction to be applied because of the measurements made in the radial blanket is minor.

We can then evaluate the a-posteriori uncertainty $\varepsilon_{C A I}^{\prime}$ on the calculated value $C_{C A I}^{\prime}$, which takes into account the experimental information; it is defined as:

$\varepsilon_{\mathrm{CAI}}^{\prime 2}=\varepsilon_{\mathrm{CAI}}^{2}\left(1-\frac{r^{2}}{1+\left(\frac{\delta E}{\varepsilon_{\mathrm{FEF}}}\right)^{2}}\right)$

In our case, the experimental information reduces the uncertainty by a factor $\varepsilon_{C A I}^{\prime} / \varepsilon_{C A I}$, which is equal to 5.3 , leading thus to $\varepsilon_{C A I}^{\prime}=0.5 \%$.

If for some reason the experimental uncertainty in the radial blanket is higher, the uncertainty reduction factor is much lower. For instance, with a $1 \%$ experimental uncertainty it drops to 3.3.

\section{Study of the ${ }^{240} \mathrm{Pu}$ production}

We can use the same method as developed previously to study the ${ }^{240} \mathrm{Pu} /{ }^{238} \mathrm{U}$ ratio, as the final amount of ${ }^{240} \mathrm{Pu}$ in the axial blankets may be of interest too. This nuclide is produced in small amount - 30 times less than the ${ }^{239} \mathrm{Pu}$ - by a capture reaction on 
Table 3

Variance of the relative uncertainty of the ${ }^{240} \mathrm{Pu} \alpha$-decay constant.

\begin{tabular}{llll}
\hline & $\mathrm{T}(\mathrm{s})$ & $\delta \mathrm{T}(\mathrm{s})$ & $\left(\frac{\delta T}{T}\right)^{2}\left(\%^{2}\right)$ \\
\hline${ }^{240} \mathrm{Pu}(\alpha)$ & $2.07 \cdot 10^{11}$ & $1.58 \cdot 10^{8}$ & $5.80 \cdot 10^{-3}$ \\
\hline
\end{tabular}

Table 4

Representativity factors for the depletion calculations of the ${ }^{240} \mathrm{Pu} /{ }^{238} \mathrm{U}$ ratio and apriori uncertainties due to nuclear data.

\begin{tabular}{llll}
\hline $1 / 2$ & $\mathrm{r}_{1,2}$ & $\varepsilon_{1}$ & $\varepsilon_{2}$ \\
\hline FEF/CAS & 0.9872 & $9.68 \%$ & $9.27 \%$ \\
FEG/CAS & 0.9998 & $9.37 \%$ & $9.27 \%$ \\
FEF/CAI & 0.9858 & $9.68 \%$ & $9.30 \%$ \\
FEG/CAI & 0.9996 & $9.37 \%$ & $9.30 \%$ \\
\hline
\end{tabular}

the ${ }^{239} \mathrm{Pu}$, so we expect a high representativity factor on the ${ }^{240} \mathrm{Pu}$ production in the various fertile zones of the reactor as well.

In the case of the ${ }^{240} \mathrm{Pu}$, we must pay attention to the following points:

- the covariance matrix $D$ now includes four isotopes: ${ }^{238} \mathrm{U},{ }^{239} \mathrm{~Np}$, ${ }^{239} \mathrm{Pu}$ and ${ }^{240} \mathrm{Pu}$. The number of entries thus increases from $200^{2}$ to $267^{2}$,

- an additional diagonal entry corresponds to the square of the relative uncertainty of the ${ }^{240} \mathrm{Pu} \alpha$-decay constant. By applying formula (6), we obtain the value for $D_{267,267}$ in Table 3 .

- as previously, we use the sensitivities of the ${ }^{238} \mathrm{U}$ and ${ }^{240} \mathrm{Pu}$ productions provided by ERANOS and we apply Eq. (2) in order to obtain the sensitivities of the ${ }^{240} \mathrm{Pu} /{ }^{238} \mathrm{U}$ ratio:

$S_{\left(\frac{\text { Pu240 }}{U 238}\right)}^{\sigma}=S_{\text {Pu240 }}^{\sigma}-S_{U 238}^{\sigma}$

The results of the representativity factors calculations are shown in Table 4.

Again, we observe very high representativity factors, which show that the ${ }^{240} \mathrm{Pu}$ production calculation is also relevant in the axial blankets of the fuel assemblies. In this case also, the experimental information in the radial blanket may be used for transposition and uncertainty reduction in the axial zones. We have higher uncertainties due to nuclear data than in the case of ${ }^{239} \mathrm{Pu}$ though, because of higher uncertainties on the ${ }^{240} \mathrm{Pu}$ production cross sections.

\section{Conclusion}

We have used the representativity theory in order to correlate the available experimental information in the radial blanket of the PHENIX reactor to our depletion calculations in the axial zones. The representativity factors that we calculate between the final ${ }^{239} \mathrm{Pu} /{ }^{238} \mathrm{U}$ ratio in the radial and axial fertile zones are very close to 1 . Hence, the measurements performed in the radial zone can help us "correct" the calculation in the axial blanket and reduce its a-priori uncertainty due to nuclear data. The estimated uncertainty reduction can reach a factor 5 , since the experimental uncertainty is low. The ${ }^{240} \mathrm{Pu} /{ }^{238} \mathrm{U}$ ratio is studied with a similar method.

\section{Acknowledgments}

The authors wish to acknowledge the financial support provided by AREVA-NC.

\section{References}

Archier, P., De Saint Jean, C., Noguère, G., Litaize, O., Leconte, P., Bouret, C., 2014. COMAC: Nuclear data covariance matrices library for reactor applications. In: PHYSOR 2014 - The Role of Reactor Physics toward a Sustainable Future Kyoto, Japan, September 28 - October 3.

Dos Santos, N., Blaise, P., Santamarina, A., 2013a. A global approach of the representativity concept: application on a high-conversion light water reactor MOX lattice case. In: Proceedings of M\&C 2013, Sun Valley, Idaho, May 5-9.

Dos Santos, N., Archier, P., Blaise, P., Santamarina, A., 2013b. Impact of mock-up experimental correlations and uncertainties in the transposition process. In: Proceedings of the 3rd International Conference on Advancements in Nuclear Instrumentation, Measurement Methods and their Applications (ANIMMA 2013).

Gandini, A. 1983. On transposition of experimental reactor data to reference design Technical report. In: Comitato nazionale per la ricerca e per lo sviluppo dell'energia nucleare e delle energie alternative.

The JEFF-3.1.1 "Nuclear Data Library JEFF Report 22", 2009. <http://www.oecd-nea. org/dbdata/nds_jefreports/jefreport-22/nea6807-jeff22.pdf>.

Kallfelz, J.M., Bruna, G.B., Palmiotti, G., Salvatores, M., 1997. Burnup calculations with time-dependent generalized perturbation theory. Nucl. Sci. Eng. 62, $304-$ 309.

Lebrat, J.F., Tommasi, J., Pascal, V., Eschbach, R., 2015. Analysis of the Trapu and Doublon irradiations in PHENIX for the experimental validation of the DARWIN package for fast reactors. In: Proceedings of the GLOBAL'2015 International Conference.

Orlov, V.V., 1980. Problems of fast reactor physics related to breeding. At. Energy Rev. 184, 989-1077.

Ruggieri, J.M.. Tommasi, J., Lebrat, J.F., 2006. ERANOS-2.1: The international code system for GEN-IV fast reactor analysis. In: Proc. Int. Conf. ICAPP'06, June 4-8, 2006, Reno, Nevada.

Tsilanizara, A. et al., 2000. DARWIN: An evolution code system for a large range of applications. J. Nucl. Sci. Technol. (Suppl 1), 845-849.

Williams, M.L., 1978. Development of depletion perturbation theory for coupled neutron/nuclide fields. Nucl. Sci. Eng. 70, 20-36.

Williams, M.L., 1986. Perturbation theory for nuclear reactor analysis. In: Ronen, Y. (Ed.), CRC Handbook of Nuclear Reactor. 